PROCEEDINGS OF THE

AMERICAN MATHEMATICAL SOCIETY

Volume 131, Number 3, Pages 793-800

S 0002-9939(02)06691-1

Article electronically published on July 2, 2002

\title{
THE BEREZIN TRANSFORM AND RADIAL OPERATORS
}

\author{
NINA ZORBOSKA \\ (Communicated by David R. Larson)
}

\begin{abstract}
We analyze the connection between compactness of operators on the Bergman space and the boundary behaviour of the corresponding Berezin transform. We prove that for a special class of operators that we call radial operators, an oscilation criterion is a sufficient condition under which the compactness of an operator is equivalent to the vanishing of the Berezin transform on the unit circle. We further study a special class of radial operators, i.e., Toeplitz operators with a radial $L^{1}(\mathbb{D})$ symbol.
\end{abstract}

Let $L_{a}^{2}$ denote the Bergman space of functions analytic on the unit disk $\mathbb{D}$. For a general bounded operator $A$ on the Bergman space, the Berezin transform of $A$ is the function $\tilde{A}$ defined by

$$
\tilde{A}(z)=\left\langle A k_{z}, k_{z}\right\rangle, \quad z \in \mathbb{D},
$$

where $\langle\cdot, \cdot\rangle$ denotes the inner product in $L_{a}^{2}$, and $k_{z}$ is the normalized evaluation function from $L_{a}^{2}$. The function $k_{z}$ is defined by

$$
k_{z}(w)=\frac{1-|z|^{2}}{(1-\bar{z} w)^{2}}, \quad \text { for } w \in \mathbb{D},
$$

and has the property that $\left\langle f, k_{z}\right\rangle=\left(1-|z|^{2}\right) f(z)$, for every $f$ in $L_{a}^{2}$.

The Berezin transform is a function that is bounded by the norm of the operator. It is also easy to see that each bounded operator on $L_{a}^{2}$ is uniquely determined by its Berezin transform. Thus, the behavior of the operator can be analyzed by exploring the corresponding Berezin transform. This idea can be employed in a much more general context where the space on which the operator acts is a so-called standard functional Hilbert space, an example of which is the Bergman space. For results on Berezin transforms on standard functional Hilbert spaces, see for example [6] and 10].

Let $P$ denote the Bergman projection from $L^{2}(\mathbb{D})$ onto $L_{a}^{2}$ and let $f \in L^{1}(\mathbb{D})$. The Toeplitz operator $T_{f}$ is defined by

$$
T_{f} g=P(f g), \quad \text { for } g \text { in } L_{a}^{2} .
$$

The Toeplitz operator is bounded whenever $f \in L^{\infty}(\mathbb{D})$, but is not bounded for every $f$ in $L^{1}(\mathbb{D})$. The boundedness and compactness of Toeplitz operators on the Bergman space have been of interest to mathematicians working in operator

Received by the editors February 6, 2001 and, in revised form, October 12, 2001.

2000 Mathematics Subject Classification. Primary 47B37, 47B10; Secondary 47B35.

Key words and phrases. Berezin transform, radial operator, Toeplitz operator.

This work was supported by an NSERC grant. 
theory and the theory of holomorphic spaces for many years. There have been many interesting results and approaches, as for example in [2], [3], [4], [9], [15] and [16.

In this article we are interested in the Berezin transform approach to compactness of radial operators motivated by the results obtained by a similar approach in the case of Toeplitz operators.

The Berezin transform of an operator $A$ on the Bergman space has an explicit formula given by

$$
\tilde{A}(z)=\left(1-|z|^{2}\right)^{2} \sum_{m, n=0}^{\infty} \sqrt{n+1} \sqrt{m+1}\left\langle A e_{n}, e_{m}\right\rangle \bar{z}^{n} z^{m}, \text { for } z \in \mathbb{D},
$$

where the functions $e_{n}(z)=\sqrt{n+1} z^{n}, n=0,1,2, \ldots$, form the standard orthonormal basis for the Bergman space. The formula for $\tilde{A}$ is derived by using the fact that the evaluation function $k_{z}$ can be expressed as a double sum over an orthonormal basis, i.e.,

$$
k_{z}(w)=\left(1-|z|^{2}\right) \sum_{m, n=0}^{\infty} \overline{e_{n}(z)} e_{n}(w), \text { for } w \in \mathbb{D} .
$$

For more details on this and on some of the properties of the Berezin transform mentioned below, see [3], [12], [16].

It is not hard to see that the Berezin transform is a real analytic function on $\mathbb{D}$, which implies that it is infinitely differentiable on $\mathbb{D}$. On the other hand, its boundary behavior can be very irregular: for example, it is not known if the Berezin transform of a bounded operator on $L_{a}^{2}$ must have radial limits almost everywhere on the unit circle. One of the deeper results on Berezin transforms obtained in [1] and [4] states that a function $f$ is a bounded harmonic function on $\mathbb{D}$ if and only if $f(z)=\widetilde{T_{f}}(z)$, for every $z \in \mathbb{D}$. Note that bounded harmonic functions have radial limits almost everywhere on the unit circle. It is also true that if $f$ is a function that is continuous on the closed unit disk, then $f(\xi)=\widetilde{T_{f}}(\xi)$ for every $\xi$ in the unit circle. See [16] for a proof of that claim.

We know more about the boundary values of the Berezin transform in the case when the corresponding operator is compact. Since $\left\{k_{z}\right\}$ converges weakly and uniformly to zero as $|z|$ converges to $1, \widetilde{A}(z)$ converges to zero uniformly as $|z|$ approaches 1 , whenever the operator $A$ is compact on $L_{a}^{2}$. The main topic in this paper is the problem of determining for which operators on $L_{a}^{2}$ the vanishing on the boundary of $\mathbb{D}$ of the Berezin transform of the operator implies that the operator is compact. This is not true for every operator, and there are several counterexamples in the literature. We will mention here two of them; both are examples of radial operators for which we provide a definition later in the paper.

One example (from [3]) is an operator that has a diagonal representation with respect to the standard basis $\left\{e_{n}\right\}$ of $L_{a}^{2}$. That operator is the composition operator $C_{\phi}$ defined by $C_{\phi} f=f \circ \phi$ for every $f$ in $L_{a}^{2}$, where $\phi(z)=-z$. The Berezin transform of $C_{\phi}$ is the function

$$
\widetilde{C_{\phi}}(z)=\frac{\left(1-|z|^{2}\right)^{2}}{\left(1+|z|^{2}\right)^{2}}
$$

and $\lim _{|z| \rightarrow 1} \widetilde{C_{\phi}}(z)=0$. On the other hand, $C_{\phi}$ is a unitary operator such that $C_{\phi}^{*}=C_{\phi}=C_{\phi}^{-1}$ and so it is not compact. 
We mention here that composition operators provide an example of a class of operators for which the Berezin transform has even more explicit form than in the general case. Namely, using the fact that $C_{\phi}^{*} k_{z}=\frac{1-|z|^{2}}{1-|\phi(z)|^{2}} k_{\phi(z)}$, we get that $\widetilde{C_{\phi}}(z)=\left(\frac{1-|z|^{2}}{1-\bar{z} \phi(z)}\right)^{2}$, for $z \in \mathbb{D}$.

Since the set of boundary fixed points of $\phi$ must have measure zero whenever $\phi$ is not the identity function, it is easy to see that $\widetilde{C_{\phi}}$ has a radial limit zero almost everywhere on the unit circle.

An additional example of a non-compact operator with boundary vanishing Berezin transform is the projection operator $P$ onto the subspace generated by the orthonormal set $\left\{e_{2^{n}}: n=0,1,2, \ldots\right\}$ (see [2]). Since the projection has an infinite dimensional rank, it is not compact, yet the Berezin transform

$$
\widetilde{P}(z)=\left(1-|z|^{2}\right)^{2} \sum_{n=0}^{\infty}\left(2^{n}+1\right)\left(|z|^{2}\right)^{2 n}
$$

converges to zero as $|z|$ converges to one.

We denote by $\vartheta$ the class of bounded operators on $L_{a}^{2}$ for which the vanishing of the Berezin transform on the boundary of the unit disk implies that the operator is compact. The previous two examples show that $\vartheta$ does not contain all of the bounded operators on $L_{a}^{2}$. In [2], S. Axler and D. Zheng have proven that finite sums of finite products of Toeplitz operators with $L^{\infty}(\mathbb{D})$ symbols belong to the class $\vartheta$. The problem of determining if this is true even for a single such Toeplitz operator has been open for a number of years. There were several partial results with special restrictions on the symbol $f$ of the Toeplitz operator $T_{f}$, under which it has been determined that $T_{f}$ must belong to the class $\vartheta$. Zhu in [15] and Luecking in [8] proved that $T_{f}$ is in $\vartheta$ whenever $f$ is bounded and positive, Korenblum and Zhu proved in [7] that the same holds if $f$ is bounded and radial, and Stroethoff proved in [13] that the same conclusion holds if $f$ is uniformly continuous with respect to the hyperbolic metric.

Our main result determines another family of operators which belong to the class $\vartheta$, namely the class of radial operators with bounded oscillation (to be defined below). These are not all Toeplitz operators and include some of the Toeplitz operators with unbounded radial symbols. For related results on Toeplitz operators with unbounded radial symbols, see [5] and [14].

Let $f$ be a function in $L^{1}(\mathbb{D})$ and let $\operatorname{rad}(f)$ be the function defined by

$$
\operatorname{rad}(f)(z)=\frac{1}{2 \pi} \int_{0}^{2 \pi} f\left(e^{i t} z\right) d t
$$

We say that $\operatorname{rad}(f)$ is the radialization of $f$, and we will say that $f$ is radial if it is equal to its radialization. Thus a radial function is such that $f(z)=f(|z|)$. Generalizing the idea to operators, for a bounded operator $A$ on $L_{a}^{2}$, we define $\operatorname{Rad}(A)$ to be the operator

$$
\operatorname{Rad}(A)=\frac{1}{2 \pi} \int_{0}^{2 \pi} U_{t}^{*} A U_{t} d t
$$


where $U_{t}$ is the unitary operator such that $\left(U_{t} f\right)(z)=f\left(e^{-i t} z\right)$ for $f$ in $L_{a}^{2}$ and $z$ in $\mathbb{D}$. The integral definition above means that for $f$ and $g$ in $L_{a}^{2}$,

$$
\langle\operatorname{Rad}(A) f, g\rangle=\frac{1}{2 \pi} \int_{0}^{2 \pi}\left\langle U_{t}^{*} A U_{t} f, g\right\rangle d t .
$$

We will say that the operator $A$ is radial whenever $A=\operatorname{Rad}(A)$. Before we provide examples of radial operators, we look more closely at the Berezin transform of $\operatorname{Rad}(A)$.

Proposition 0.1. $\widetilde{\operatorname{Rad}(A})(z)=\operatorname{Rad}(\widetilde{A})(z)$ for all $z$ in the unit disk, and $A$ is radial if and only if its Berezin transform function is radial.

Proof. Since

$$
U_{t} k_{z}(w)=k_{z}\left(e^{-i t} w\right)=\frac{1-|z|^{2}}{\left(1-\bar{z} e^{-i t} w\right)^{2}}=k_{e^{i t} z}(w)
$$

we have that

$$
\begin{aligned}
\widetilde{\operatorname{Rad}(A)}(z) & =\left\langle\operatorname{Rad}(A) k_{z}, k_{z}\right\rangle=\frac{1}{2 \pi} \int_{0}^{2 \pi}\left\langle A U_{t} k_{z}, U_{t} k_{z}\right\rangle d t \\
& =\frac{1}{2 \pi} \int_{0}^{2 \pi}\left\langle A k_{e^{i t} z}, k_{e^{i t} z}\right\rangle d t=\frac{1}{2 \pi} \int_{0}^{2 \pi} \widetilde{A}\left(e^{i t} z\right) d t .
\end{aligned}
$$

Thus $\widetilde{\operatorname{Rad}(A)}(z)=\operatorname{rad}(\widetilde{A})(z)$ for all $z$ in $\mathbb{D}$. Hence, if $A$ is a radial operator, then its Berezin transform must be a radial function. On the other hand, whenever the Berezin transform of an operator is radial, i.e., whenever $\widetilde{\operatorname{Rad}(A)}(z)=\widetilde{A}(z)$ for all $z$ in $\mathbb{D}$, since the operators are uniquely determined by their Berezin transform, $\widetilde{\operatorname{Rad}(A)}=\widetilde{A}$ implies that $\operatorname{Rad}(A)=A$.

Now, for $f$ in $L^{1}(\mathbb{D})$, let

$$
\tilde{f}(z)=\int_{\mathbb{D}} f(w)\left|k_{z}(w)\right|^{2} d m(w)
$$

Even though the Toeplitz operator $T_{f}$ may not be bounded, its domain includes $H^{\infty}$ and its Berezin transform $\widetilde{T_{f}}$ is well defined and equal to $\tilde{f}$. We call $\tilde{f}$ the Berezin transform of the function $f$. Note that $\widetilde{\operatorname{rad}(f)}=\operatorname{rad}(\widetilde{f})$, since for $z$ in $\mathbb{D}$, we have

$$
\begin{aligned}
\widetilde{\operatorname{rad}(f)}(z) & =\int_{\mathbb{D}} \operatorname{rad}(f)(w)\left|k_{z}(w)\right|^{2} d m(w) \\
& =\frac{1}{2 \pi} \int_{0}^{2 \pi}\left(\int_{\mathbb{D}} f\left(w e^{i t}\right)\left|k_{z}(w)\right|^{2} d m(w)\right) d t \\
& =\frac{1}{2 \pi} \int_{0}^{2 \pi}\left(\int_{\mathbb{D}} f\left(w e^{i t}\right)\left|k_{e^{i t} z}\left(e^{i t} w\right)\right|^{2} d m(w)\right) d t \\
& =\frac{1}{2 \pi} \int_{0}^{2 \pi}\left(\int_{\mathbb{D}} f(u)\left|k_{e^{i t} z}(u)\right|^{2} d m(u)\right) d t \\
& =\frac{1}{2 \pi} \int_{0}^{2 \pi} \tilde{f}\left(e^{i t} z\right) d t=\operatorname{rad}(\tilde{f})(z) .
\end{aligned}
$$

Thus, $f$ is radial if and only if $\tilde{f}$ is radial and so, by Proposition 0.1, the Toeplitz operator $T_{f}$ is radial if and only if $f$ is radial. 
Note also that every operator $A$ with harmonic Berezin transform must be a Toeplitz operator with symbol $\widetilde{A}$. That is true since $\widetilde{A}=\tilde{\tilde{A}}=\widetilde{T_{\widetilde{A}}}$. So, a radial operator with harmonic Berezin transform is a Toeplitz operator $T_{f}$ with radial harmonic symbol $f$. But, for such an operator, since $\lim _{r \rightarrow 1} f(r)$ must exist, i.e., since $f$ is continuous on the closed unit disk, the operator $T_{f}$ is compact if and only if $f(1)=0$.

Proposition 0.2. Let $A$ be a radial bounded operator on $L_{a}^{2}$. Then $A$ is a diagonal operator with respect to the standard basis $\left\{e_{n}\right\}$ of $L_{a}^{2}$.

Proof. This is easy to see from the following:

$$
\begin{aligned}
\left\langle A e_{n}, e_{m}\right\rangle & =\left\langle\operatorname{RadA} e_{n}, e_{m}\right\rangle=\frac{1}{2 \pi} \int_{0}^{2 \pi}\left\langle A U_{t} e_{n}, U_{t} e_{m}\right\rangle d t \\
& =\frac{1}{2 \pi} \int_{0}^{2 \pi}\left\langle A\left(e^{-i n t}\right), e^{-i n t} e_{m}\right\rangle d t \\
& =\frac{1}{2 \pi} \int_{0}^{2 \pi} e^{-i(m-n) t}\left\langle A e_{m}, e_{n}\right\rangle d t=0, \text { whenever } n m
\end{aligned}
$$

Note that the compactness of an operator $A$ on the Bergman space can be expressed with the help of radial operators as follows: $A$ is compact if and only if $A^{*} A$ is compact if and only if there exists a unitary operator $U$ such that $U^{*} A^{*} A U$ is a compact radial operator.

Let $a_{n}=\left\langle A e_{n}, e_{n}\right\rangle$. We want to see what the correlation is between the behavior of the sequence $\left\{a_{n}\right\}$ and the boundary behaviour of the Berezin transform $\tilde{A}$. Of course, whenever $a_{n}$ converges to zero, if $|z|$ approaches one, then $\tilde{A}(z)$ converges to zero. The converse is not always true, as the two counterexamples of diagonal non-compact operators above show. We will show that under a specific oscillation restriction on the sequence $\left\{a_{n}\right\}$ we have that for the radial operator $A, \tilde{A}(z) \rightarrow 0$ as $|z| \rightarrow 1$ is a sufficient condition for compactness of $A$, i.e., that $A$ belongs to the class $\vartheta$.

Note first that for radial $A$, since $\tilde{A}(z)=\operatorname{rad}(\tilde{A})(z)=\tilde{A}(|z|)$, we have that $\tilde{A}(z)=\left(1-|z|^{2}\right)^{2} \sum_{n}(n+1) a_{n}|z|^{2 n}$. Thus, the question of whether $A$ is in $\vartheta$ is equivalent to the following problem:

- when does $\lim _{|t| \rightarrow 1^{-}}\left(1-|z|^{2}\right)^{2} \sum_{n}(n+1) a_{n}|z|^{2 n}=0$ imply that $a_{n} \rightarrow 0$ as $n \rightarrow \infty$ ?

In general, this is a problem on sequences and series of complex numbers and has a connection with applications of Tauberian theorems, such as the ones considered in [11. The same approach has been used in [7] to prove that in case $A$ is a Toeplitz radial operator $T_{f}$ with a bounded symbol $f$, we have that $a_{n} \rightarrow 0$, as $n \rightarrow \infty$, if and only if $\left(1-|z|^{2}\right)^{2} \sum_{n=0}^{\infty}(n+1) a_{n}|z|^{2 n} \rightarrow 0$ as $|z| \rightarrow 1$. We give below a sufficient condition for a general radial operator $A$ such that the convergence of the above series to zero as $|z| \rightarrow 1$ implies that $a_{n} \rightarrow 0$, as $n \rightarrow \infty$, i.e., that the operator $A$ is compact.

Theorem 0.3. Let $A$ be a bounded radial operator on the Bergman space with diagonal $\left\{a_{n}\right\}$, such that $n\left(a_{n}-a_{n-1}\right)$ is bounded. Then $\tilde{A}(z) \rightarrow 0$, as $|z| \rightarrow 1^{-}$, implies that $A$ is compact (i.e., $A$ belongs to the class $\vartheta$ ). 
Proof. Since $A$ is radial, $\tilde{A}(z)=\left(1-|z|^{2}\right)^{2} \sum_{n=0}^{\infty}(n+1) a_{n}|z|^{2 n}$. A result from [11] states that for a sequence $\left\{b_{n}\right\}$ such that

$$
\lim _{t \rightarrow 1^{-}}(1-t) \sum_{n=0}^{\infty} b_{n} t^{m}=0
$$

and such that $\left\{b_{n}\right\}$ is bounded, we have that

$$
\lim _{n \rightarrow \infty} \frac{1}{n+1} \sum_{k=0}^{n} b_{k}=0 .
$$

Let $b_{0}=a_{0}$, let $b_{n}=(n+1) a_{n}-n a_{n-1}$, for $n \geq 0$ and let $t=|z|^{2}$. Then by an argument similar to that in 7 we have that

$$
\begin{aligned}
\lim _{t \rightarrow 1^{-}} \tilde{A}(z) & =\lim _{t \rightarrow 1^{-}}(1-t)^{2} \sum_{n=0}^{\infty}(n+1) a_{n} t^{n} \\
& =\lim _{t \rightarrow 1^{-}}(1-t)\left(a_{0}+\sum_{n=1}^{\infty}\left((n+1) a_{n}-n a_{n-1}\right) t^{n}\right. \\
& =\lim _{t \rightarrow 1^{-}}(1-t)\left(a_{0}+\sum_{n=1}^{\infty} b_{n} t^{n}\right)=0 .
\end{aligned}
$$

Thus, $\lim _{t \rightarrow 1^{-}}(1-t)\left(\sum_{n=0}^{\infty} b_{n} t^{n}\right)=0$. We also have that $\left\{b_{n}\right\}$ is bounded, since $b_{n}=n\left(a_{n}-a_{n-1}\right)+a_{n}$, and so $\lim _{n \rightarrow \infty} \frac{1}{n+1} \sum_{k=0}^{n} b_{k}=0$. But

$$
\frac{1}{n+1} \sum_{k=0}^{n} b_{k}=\frac{1}{n+1}\left(a_{0}+\sum_{k=1}^{n}\left((k+1) a_{k}-k a_{k-1}\right)\right)=\frac{1}{n+1}(n+1) a_{n}=a_{n},
$$

and we have that $\lim _{n \rightarrow \infty} a_{n}=0$. So, the operator $A$ is compact.

Note that for a general radial operator $A$, the boundedness of $n\left(a_{n}-a_{n-1}\right)$ does not imply compactness of $A$. For example, that happens when $A$ is a scalar operator. Another counterexample is when $A$ is a diagonal operator with diagonal $\left\{a_{n}\right\}$ such that each $a_{n}$ is a point on the curve $\left(t, \sin \frac{1}{t}\right), t>0$. If each $a_{n}=\left(t_{n}\right.$, $\left.\sin \frac{1}{t_{n}}\right)$ is chosen such that $t_{n} \rightarrow 0$ as $n \rightarrow \infty$, and such that the length of the curve between $a_{n}$ and $a_{n-1}$ is $\frac{1}{n}$, then we have that the set of limit points of $\left\{a_{n}\right\}$ is the closed interval $[-1,1]$. Hence, the operator $A$ is neither compact nor is it a scalar plus a compact, even though $n\left(a_{n}-a_{n-1}\right)$ is bounded.

The condition that $n\left(a_{n}-a_{n-1}\right)$ is bounded is a criterion on the oscillation of the sequence $\left\{a_{n}\right\}$; it requires that the rate of oscillation cannot be slower than $\frac{1}{n}$. Note that as long as the oscillation of a sequence tends to zero, i.e., as long as $a_{n}-a_{n-1} \rightarrow 0$, as $n \rightarrow \infty$, we have that either the sequence converges, or that the sequence has an uncountable set of accumulation points. In fact, as pointed out in [5], it is easy to see that whenever $a_{n}-a_{n-1} \rightarrow 0$, as $n \rightarrow \infty$, the set of limit points of the sequence $\left\{a_{n}\right\}$ must be a closed connected subset of $\mathbb{C}$. If that set is a single point, i.e., if the sequence $\left\{a_{n}\right\}$ converges, then the operator $A$ is a sum of a scalar and a compact operator. That happens, for example, whenever $A$ is a radial Toeplitz operator $T_{f}$ with $f$ continuous on the closed unit disk, i.e., with $f$ such that $\lim _{r \rightarrow 1^{-}} f(r)$ exists. It can also happen even when $f$ is unbounded and with $\lim _{r \rightarrow 1^{-}} f(r)$ not existing, since the operator $T_{f}$ can still be compact in that case. Recall that if $T_{f}$ is compact, then $\lim _{r \rightarrow 1^{-}} \widetilde{f(r)}=\lim _{n \rightarrow \infty} a_{n}=0$, but 
for unbounded $f$, it is not known if $\lim _{r \rightarrow 1} \widetilde{f(r)}=0$ implies that $\lim _{n \rightarrow \infty} a_{n}=0$. The above proposition guarantees that if the oscillation condition is satisfied, then $T_{f}$ is in the class $\vartheta$ even though $f$ is unbounded, i.e., $\tilde{f}(z) \rightarrow 0$ if and only if $\lim _{n \rightarrow \infty} a_{n}=0$.

Theorem 0.4. Let $f$ be a radial function in $L^{1}(\mathbb{D})$ and let $T_{f}$ be bounded on $L_{a}^{2}$. If $f(r)-\frac{1}{1-r} \int_{r}^{1} f(s) s d s$ is bounded for $0 \leq r<1$, then $T_{f}$ is compact if and only if $\lim _{r \rightarrow 1^{-}} \widetilde{f(r)}=0$, and so $T_{f}$ is in the class $\vartheta$.

Proof. If $A$ is the Toeplitz operator $T_{f}$, then

$$
a_{n}=(n+1) \int_{\mathbb{D}} f(z)|z|^{2 n} d m(z) .
$$

So, if $f$ is radial, i.e., if $f(z)=f(|z|)$, changing to polar coordinates, we get that

$$
a_{n}=2(n+1) \int_{0}^{1} f(r) r^{2 n+1} d r
$$

But then $n\left(a_{n}-a_{n-1}\right)=2 n^{2} \int_{0}^{1} f(r) r^{2 n-1}\left(1-r^{2}\right) d r+2 n \int_{0}^{1} f(r) r^{2 n+1} d r$. The second term in the sum equals $\frac{n}{n+1} a_{n}$, and so, for a bounded operator $T_{f}$ we have that $n\left(a_{n}-a_{n-1}\right)$ is bounded whenever the first term in the sum is bounded. On the other hand

$$
\begin{aligned}
& 2 n^{2} \int_{0}^{1} f(r) r^{2 n-1}\left(1-r^{2}\right) d r \\
= & 2 n^{2} \int_{0}^{1}\left(f(r)-\frac{1}{1-r} \int_{r}^{1} f(s) s d s\right) r^{2 n-1}\left(r^{2}-1\right) d r \\
& +2 n^{2} \int_{0}^{1} \frac{1}{1-r}\left(\int_{r}^{1} f(s) s d s\right) r^{2 n-1}\left(r^{2}-1\right) d r .
\end{aligned}
$$

Using integration by parts in the second summand we can see that it has the same rate of growth as $a_{n}$, i.e., that it is bounded. Since $2 n^{2} \int_{0}^{1} r^{2 n-1}\left(r^{2}-1\right) d r \leq 2$, we get that $n\left(a_{n}-a_{n-1}\right)$ is bounded whenever $f(r)-\frac{1}{1-r} \int_{r}^{1} f(s) s d s$ is bounded for $0 \leq r<1$.

Note that in case $f$ is radial and is in $L^{\infty}(\mathbb{D})$, we get that $f(r)-\frac{1}{1-r} \int_{r}^{1} f(s) s d s$ is also bounded, and so, by Theorem $0.4, T_{f}$ is compact if and only if $\lim _{r \rightarrow 1^{-}} \widetilde{f}(r)=0$. The fact that $T_{f}$ is compact if and only if $\lim _{r \rightarrow 1^{-}} \widetilde{f}(r)=0$ whenever $f$ is bounded (and radial) was originally proved in [7].

Note also that in case $f$ is radial and positive, the expression $\frac{1}{1-r} \int_{r}^{1} f(s) s d s$ plays a special role in the so-called Carleson measure characterization of the boundedness and compactness of $\mathrm{T}_{f}$.

Let $S_{\xi}(r)=\left\{z \in \mathbb{D} ; 1-r<|z|<1, \arg \xi-\frac{1-r}{2}<\arg z<\arg \xi+\frac{1-r}{2}\right\}$ for $0 \leq$ $r<1$ and $\xi$ in the unit circle $\partial \mathbb{D}$. D. Luecking's results from [7] tell us that for $f$ positive and in $L^{1}(\mathbb{D})$, the operator $T_{f}$ is bounded on $L_{a}^{2}(\mathbb{D})$ if and only if $\frac{1}{m\left(S_{\xi}(r)\right)} \int_{S_{\xi}(r)} f(z) d m(z)$ is bounded for all $\xi$ in the unit circle $\partial \mathbb{D}$ and all $0 \leq r<1$; the operator $T_{f}$ is compact if and only if

$$
\lim _{r \rightarrow 1} \sup _{\xi \in \partial \mathbb{D}} \frac{1}{m\left(S_{\xi}(r)\right)} \int_{S_{\xi}(r)} f(z) d m(z)=0 .
$$


Since $m\left(S_{\xi}(r)\right)$ is equivalent to $\frac{1}{(1-r)^{2}}$, then, whenever $f$ is positive and radial, we have that

$$
\frac{1}{m\left(S_{\xi}(r)\right)} \int_{S_{\xi}(r)} f(z) d m(z)=\frac{1}{m\left(S_{1}(r)\right)} \int_{S_{1}(r)} f(z) d m(z)=\frac{1}{\pi} \frac{1}{1-r} \int_{r}^{1} f(s) s d s .
$$

Hence, the boundedness and compactness of $T_{f}$ on $L_{a}^{2}(\mathbb{D})$ for $f$ positive and radial can be restated in terms of $\frac{1}{1-r} \int_{r}^{1} f(s) s d s$. Similar results can also be found in [5] and 14 .

\section{REFERENCES}

[1] Ahern, P., Flore, M., and Rudin, W.: An Invariant Volume-Mean-Value Property, J. Funct. Anal. 111 (1993), 380-397. MR 94b:31002

[2] Axler, S., Zheng, D.: Compact Operators via the Berezin Transform, Indiana University Mathematics Journal 47 (1998), 387-400 MR 99i:47045

[3] Axler, S., Zheng, D.: The Berezin Transform on the Toeplitz Algebra, Studia Mathematica 127 (1998), 113-136 MR 98m:47030

[4] Englis, M.: Functions Invariant Under the Berezin Transform, J. Funct. Anal. 121 (1994), 223-254 MR 95h:31001

[5] Grudski, S., Vasilevski, N.: Bergman-Toeplitz Operators:Radial Component Influence, Integral Equations Operator Theory 40 (2001), no. 1, 16-33

[6] Kiliç, S.: The Berezin Symbol and Multipliers of Functional Hilbert Spaces, Proc. Amer. Math. Soc 123 (1995), 3687-3691 MR 96b:46035

[7] Korenblum, B. and Zhu, K. H.: An Application of Tauberian Theorems to Toeplitz Operators, Journal of Operator Theory 33 (1995), 353-361 MR 96i:47046

[8] Luecking, D.: Trace Ideal Criteria for Toeplitz Operators, Journal of Functional Analysis 73, (1987), 345-368 MR 88m:47046

[9] McDonald, G., Sundberg, C.:Toeplitz Operators on the Disk, Indiana University Math. J. 28 (1979), 595-611 MR 80h:47034

[10] Nordgren, E., Rosenthal, P.: Boundary Values of Berezin symbols, Operator Theory Advances and Applications 73 (1994), 362-368 MR 96b:46036

[11] Postnikov, A.G.: Tauberian Theory and its Applications, Proc. Steklov Inst. Math., Amer. Math. Soc., Vol. 144, 1980 MR 82f:40012b

[12] Stroethoff, K.: The Berezin Transform and Operators on Spaces of Analytic Functions, Banach Center Publ. 38 (1997), 361-380 MR 98g:47025

[13] Stroethoff, K.: Compact Toeplitz Operators on Bergman Spaces, Math. Proc. Cambridge Philos. Soc. 124 (1998), 151-160 MR 99i:47046

[14] Vukotić, D.: Carleson Measures and Bergman Spaces Operators, Preprint

[15] Zhu, K.: Positive Toeplitz Operators on Weighted Bergman Spaces of Bounded Symmetric Domains, J. Operator Theory 20 (1988), 329-357 MR 92f:47022

[16] Zhu, K.: Operator Theory in Function Spaces, Marcel Dekker, Inc., 1990 MR 92c:47031

Department of Mathematics, University of Manitoba, Winnipeg, Manitoba, Canada R3T 2N2

E-mail address: zorbosk@cc.umanitoba.ca 